\title{
2D Numerical Simulation of a Vacuum Assisted Resin Infusion
}

\author{
Adam Kazda ${ }^{1,{ }^{*}}$, Pavel Peukert ${ }^{1}$, and Jan Novosád ${ }^{1}$ \\ ${ }^{1}$ VÚTS, a.s., Svárovská 619, Liberec, Czech Republic
}

\begin{abstract}
The subject of this work is an application of CFD for simulation of vacuum assisted resin infusion. The microscale behaviour is neglected and fibres in the mould are replaced by the porous zone. Equations for porosity coefficients are altered so the coefficients can be computed from the experiment's outputs. The experiment is simplified by filling the mould with fibres without additional layers, which are commonly used in industry manufacturing. There are two experiments. The first one is simple and is used like an input for the simulation. Results of second one are compared with the simulation.
\end{abstract}

\section{Introduction}

Fibre-reinforced plastics are composites, which are widely used in many industries (e.g. automotive, aerospace, energetics). They consist from a polymer matrix, which is reinforced with fibres (e.g. glass, carbon, aramid, etc.). Therefore they combine beneficial properties of both phases, the lightness of plastics and strength of fibres. Reinforced plastics are manufactured in many different ways like injection, infusion and manual or spray application. Two most common methods are a resin transfer moulding (RTM) and a vacuum assisted resin transfer moulding (VARTM), also called a vacuum assisted resin infusion (VARI) [1,2].

RTM uses moulding form, which consists of two parts. These parts create a cavity of the final shape. The fibre preform is pressed by the moulding form and the cavity can be filled by resin under pressure. The moulding form for RTM is expensive, so it is mainly used in a mass production. VARI has a simpler moulding form than RTM, because it consists of only one side and another side is replaced by a vacuum bag. The disadvantage of VARI is that the filling is done by a suction of vacuum and cannot be accelerated by pressure at inlet. VARI technology is used also for larger parts like boat hulls, wind turbine blades and so on $[3,4]$.

\footnotetext{
* Corresponding author: adam.kazda@vuts.cz
} 


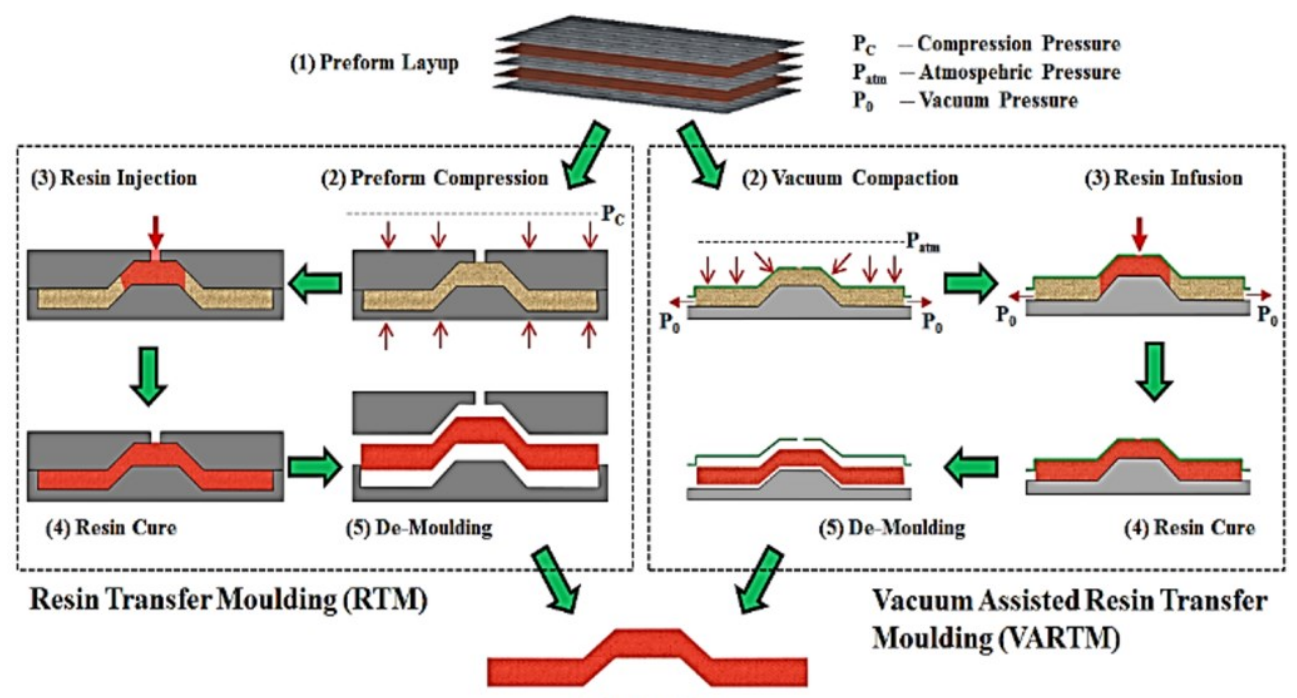

(6) Finished Part

Fig. 1. An illustration of RTM and VARTM manufacturing processes. [1]

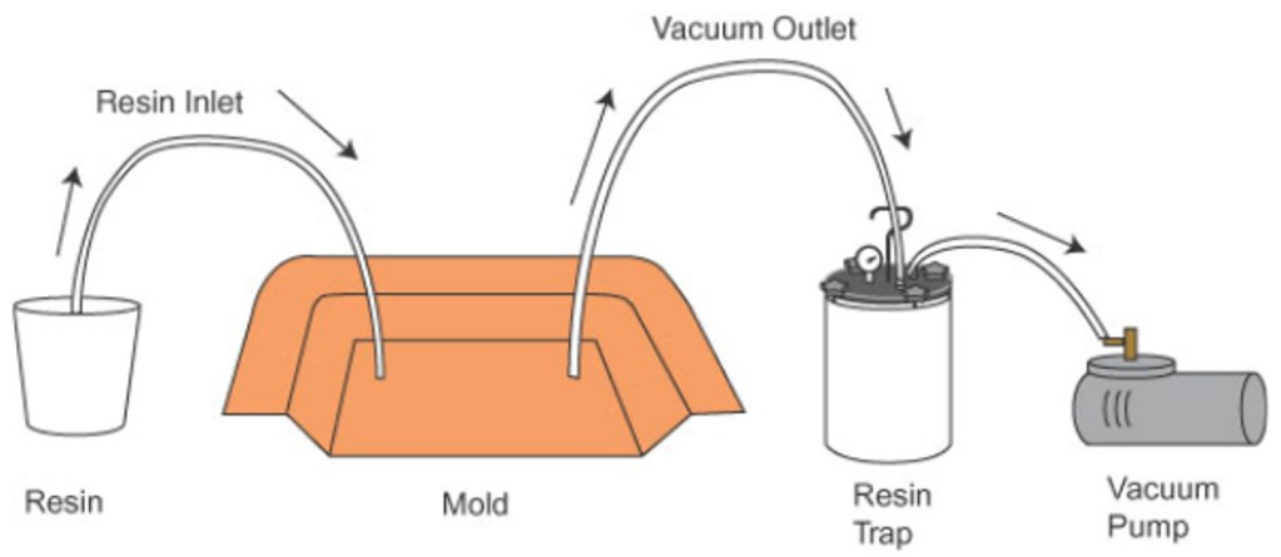

Fig. 2. Simplified depiction of a vacuum infusion process. [5]

In this article, simulations are focused on the VARI technology. A simplified depiction is shown in Figure 2. The mould is filled with fibres, release peel ply and a distribution layer. Only fibres stay in the final product. The distribution layer helps the resin to flow through the mould and in the final stage simplifies a suction of air bubbles from the laminate. The release peel ply provides an easy release barrier between the laminate surface and the distribution layer. A common defects of VARI technology are voids in the laminate. Mould filling simulations should help to avoid these defects. Benefit of the simulation increases with the rising cost of materials or dimensions of manufactured part. Simulations are also helpful for an optimization of the number of the inlets and the outlets and their positions, especially in case of complicated shapes $[2,6]$.

\section{Experiment}


The composite created in the experiment consists of epoxy resin and chopped strand mat with area density of $450 \mathrm{~g} / \mathrm{m}^{2}$. The chop strand mat is a fabric made of randomly oriented e-glass fibres. An important parameter of the resin is gel time, which defines the interval of workability. The used epoxy resin LH385 with hardener H286 has gel time of the used resin up to 90 minutes. Physical properties of the resin given by the manufacturer are: dynamic viscosity $(400-800) \mathrm{mPa} \cdot \mathrm{s}$, density $(1.12-1.16) \mathrm{g} / \mathrm{cm}^{3}$. The composite is manufactured without a distribution layer and a release peel ply in order to simplify the case for the simulation. The flow in the mould is decelerated without the distribution layer, so dimensions of the final product are limited due workability time. The vacuum pump provides $80 \mathrm{kPa}$ vacuum.

\subsection{Simple plate without an obstacle}

For the initial experiment a plate $(25 \times 25) \mathrm{cm}$ is manufactured at horizontal plane and thus obtaining input parameters for the simulation. Experiments are performed with different number of layers (2, 10 and 30) to investigate the dependency of the flow speed on the thickness of the laminate. One layer of chopped strand mat with area density $450 \mathrm{~g} / \mathrm{m}^{2}$ has thickness approximately $0.4 \mathrm{~mm}$. The evaluation of the experiment is done from top view pictures, which are taken by the camera every 10 seconds. The area filled by resin is measured in every picture. An example of the picture is shown in Figure 3. For the experiment with 30 layers an automatic evaluation is used. Other experiments have unsatisfactory light conditions, so the image evaluation is done manually. The dependency of velocity of the interface on time is shown in Figure 4. The exponential interpolation of these data is used for the porosity coefficient computation. There is also obvious that flow is independent on the number of layers. Therefore the simple evaluation of the plane can be simplified into a $1 \mathrm{D}$ case. There are only few negligible differences at the beginning.

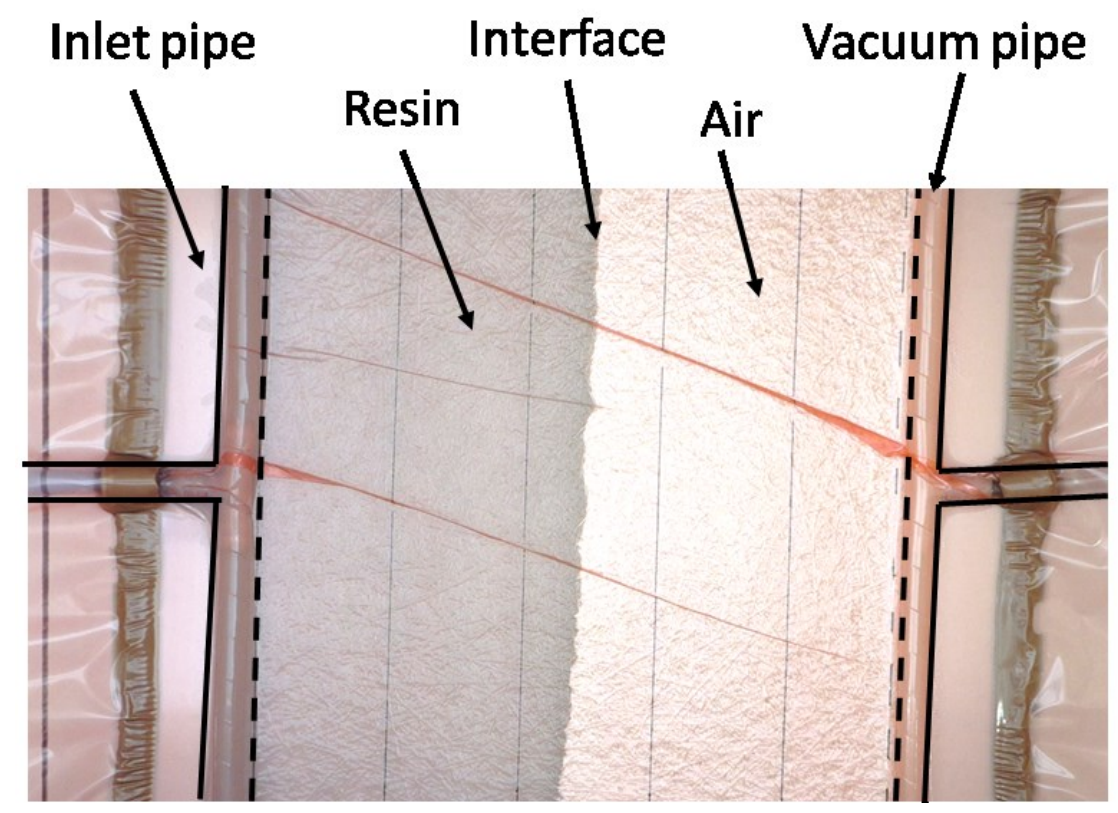

Fig. 3. Simple plate mould filling. 


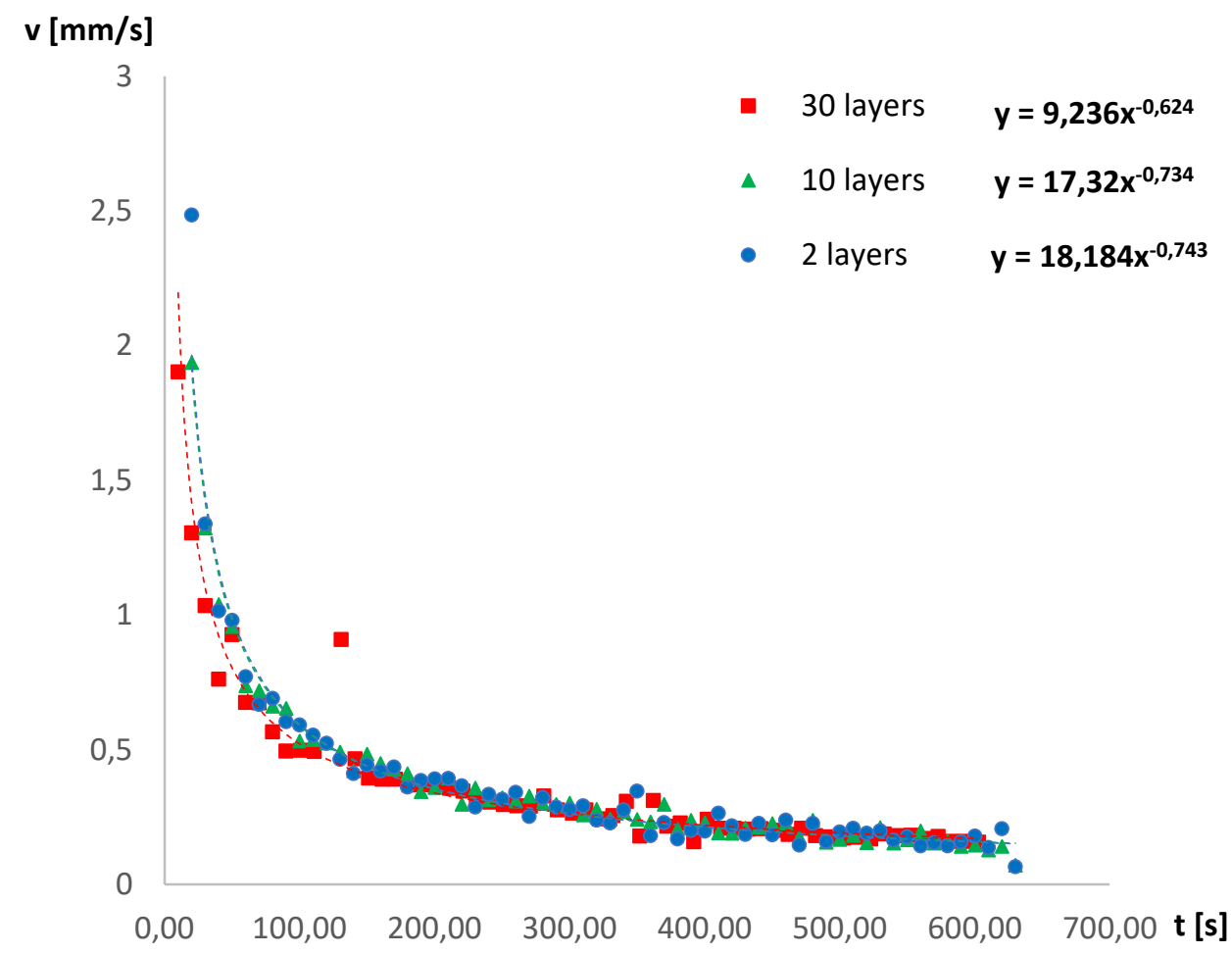

Fig. 4. Interface velocity in time.

\subsection{Plate with an obstacle}

To investigate the $2 \mathrm{D}$ behaviour of the flow in the mould a square shaped obstacle is placed into the channel. The geometry of the channel is shown in the Figure 5. The obstacle is made in two different ways, dependent on the layer count. A hole is cut into fabric in both cases. In the case with ten layers of fabric a wooden prism is placed into the hole. But for the case with just two layers the covering foil at the hole is sucked directly to the bottom of the moulding form. A phenomenon called edge flow [7] is observed in both cases. It means that the flow is accelerated close to the walls.

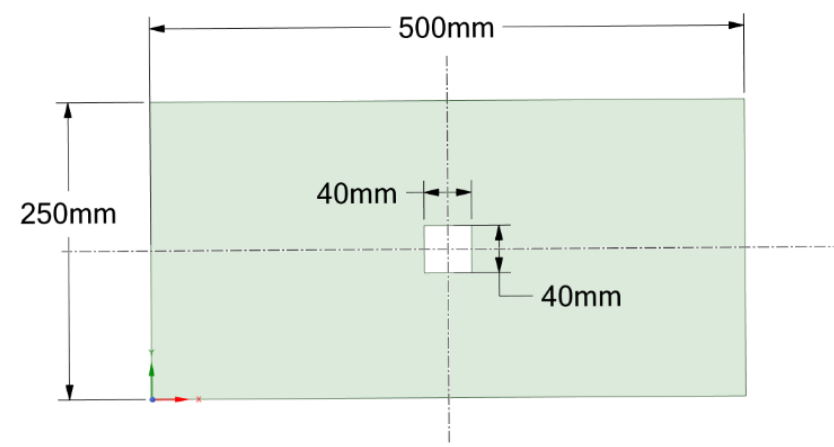

Fig. 5. Geometry with an obstacle. 




(a)

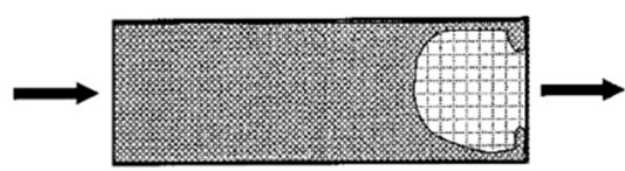

(c)



(b)



(d)

Fig. 6. Edge flow. [7]

\section{Simulation}

The Reynolds number of the flow in the moulding form is far below 1 so equations for a laminar flow can be used. Only 1D simulation is done for the simple plate where the width and the thickness of the plate are neglected. A sensitivity for the grid size is tested in this case. The fine mesh has an element size of $0.5 \mathrm{~mm}$ and the coarse one $5 \mathrm{~mm}$. Both simulations provide same results. The number of elements is 500 for fine mesh and 50 for coarse mesh. The $2 \mathrm{D}$ simulation is done for the case with obstacle, where the thickness of the laminate is neglected. In this case a mesh with an element size of $1 \mathrm{~mm}$ is used with a refinement at the rear edge of the obstacle. Fibres inside the moulding form are replaced by the porous zone. This approach neglects the microscale of fibres, but it should be suitable for macroscales.

\subsection{Porous zone}

The Darcy law is used for the porous zone which adds a momentum source term to standard fluid equations:

$$
S_{i}=-\sum_{j=1}^{2} D_{i j} \mu v_{j}
$$

where $S_{i}$ is the source term for the $\mathrm{i}$-th (x,y) momentum equation, $v$ is the velocity component, $\mu$ is the kinematic viscosity and $D$ is prescribed matrices. This source term expresses viscous losses. Inertial losses are neglected because of the laminar flow behaviour. The Equation (1) can be simplified for homogenous porous medium into:

$$
S_{i}=-\frac{\mu}{\alpha} v_{i}
$$

where $D$ is replaced by the permeability $1 / \alpha$. The pressure drop is proportional to the velocity in laminar flows through porous media so the equation can be transformed into: 


$$
\frac{\mu}{\alpha}=\frac{\Delta p}{v \cdot \Delta n},
$$

where $\Delta n$ is the thickness of the medium. The left side of the equation describes a combination of properties of the fluid and the porous zone and can be easily computed from experimental data which are at right side of the equation [6].

\section{Results}

The movement of the interface between the resin and air is used to compare between the experiment and simulation. The velocity of the interface provides also the velocity along the whole domain.

\subsection{Simple plate without an obstacle}

The velocity for the computation of the porosity coefficient is taken from the frames where the interface nearly reaches the end of the mould bag. The influence of air resistance should be minimal. The comparison of the simulation and the experiment is shown in Figure 7. The dependency received from the simulation corresponds well with the experimental results.

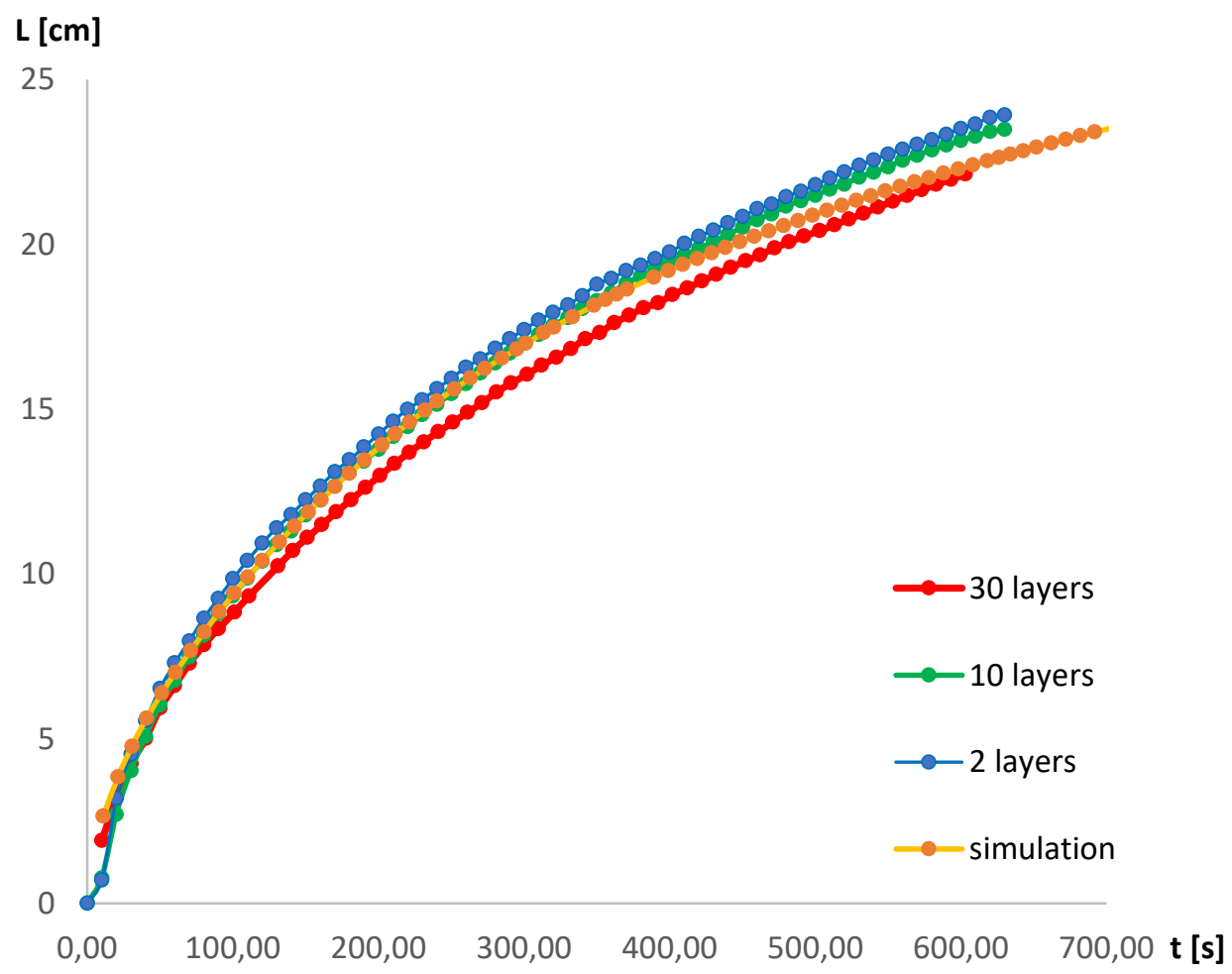

Fig. 7. Interface position in time. 


\subsection{Plate with an obstacle}

The porosity coefficient for this simulation is taken from the previous experiment of the simple plate without an obstacle, because the goal is to predict the filling behaviour of any complex mould. The comparison of the simulation and the experiment is shown in Figure 8. The simulation with the porosity from the previous simulation corresponds with the experiment until $1000 \mathrm{~s}$. Then the flow in the simulation is faster than in the experiment. Although the manufacturer states that the manufacturing time is 90 minutes the change of speed can be caused by a raising viscosity of resin. Therefore the porosity coefficient is evaluated for different time frames. A linear regression of these data is done and we get a time dependent porosity coefficient. The simulation with the time dependent porosity coefficient fits well the experiment with 10 layers.

The fitting curve is found and Figure 9 compares the resin flow progress close to the obstacle between the numerical simulation and the experiment. There is a good agreement in the position of the interface at $t=840 \mathrm{~s}$ but we can see the accelerated flow close to the walls because of the edge flow. In $t=1130 \mathrm{~s}$ there is an obvious difference. The flow in the experiment overcomes the obstacle easier due to the edge flow. In $t=1370 \mathrm{~s}$ is a velocity profile behind the obstacle in the simulation, but in the experiment the interface is already straightened.

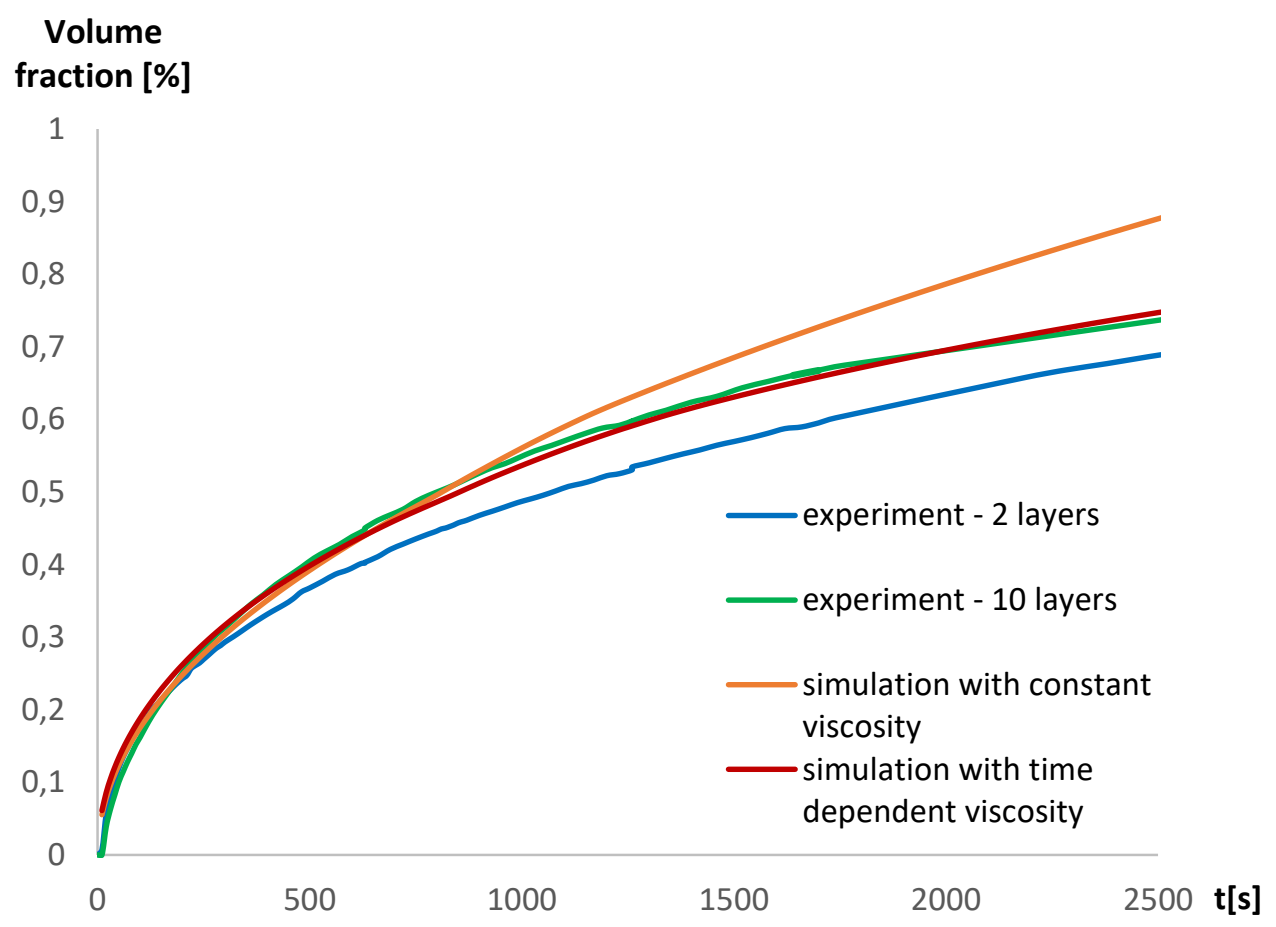

Fig. 8. Comparison of the simulation and the experiment. 


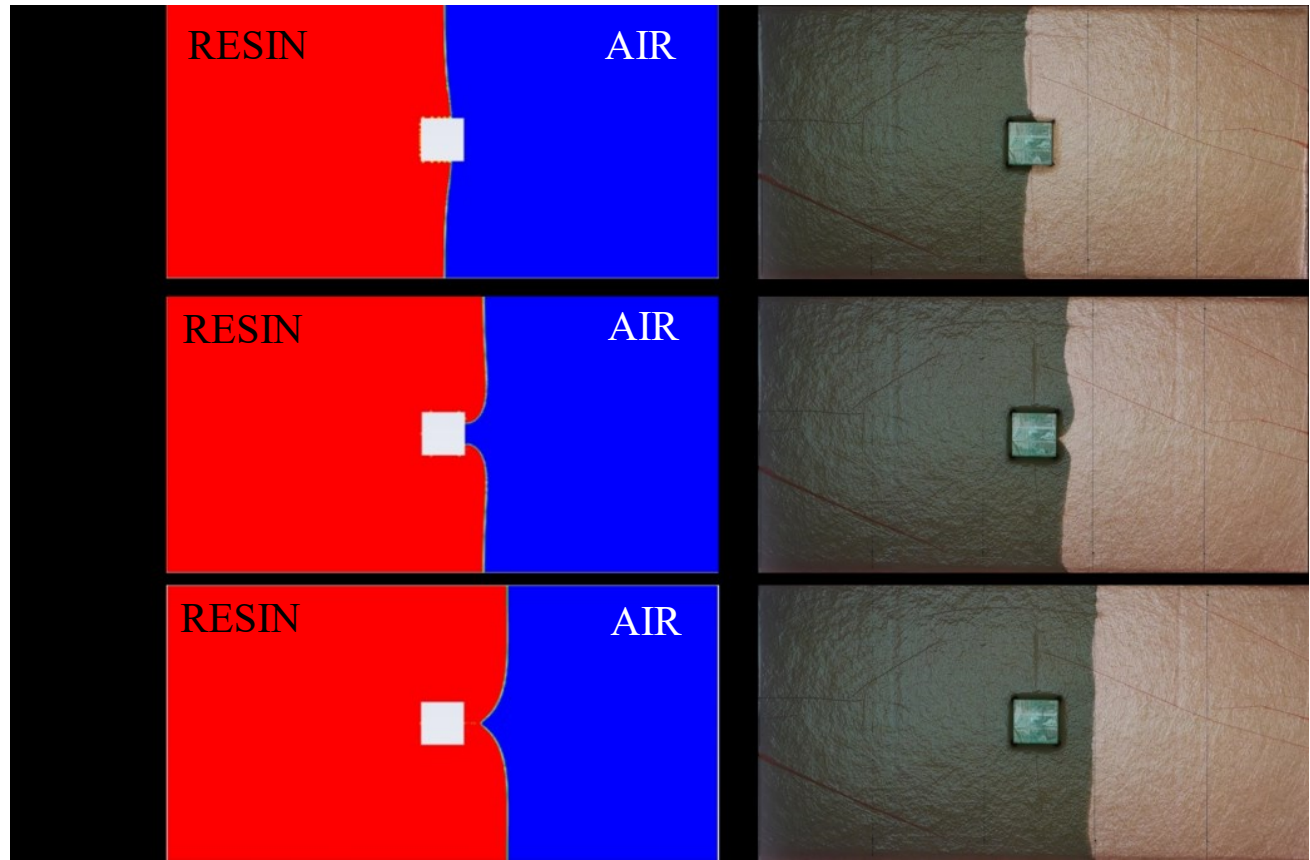

Fig. 9. Comparison of the flow field in the simulation and the experiment.

\section{Conclusion}

Numerical simulations with the use of porous media formulation for a simulation of a vacuum assisted resin infusion was investigated. A porosity coefficient was taken from the testing sample. This testing sample was done for three different thickness of the laminate. Comparing results of a flow speed for different thickness showed that the flow through the mould is independent on the thickness so the thickness can be neglected in simulations.

The application of porosity coefficient from the testing sample on larger plate with an obstacle worked just for a limited amount of time. After this time the flow in the simulation was faster than in the experiment. It is expected that this change is caused by the raising viscosity of the resin. In the simulation the porosity coefficient counts with the resistance of fibres and also the viscosity of the fluid. A simulation with a time dependent porosity coefficient, which substitutes the changing viscosity, agreed well with the experiment.

The flow field was similar in the experiment and the simulation for the flow around an obstacle. Differences were mostly caused by the edge flow which accelerates the flow along the walls. This acceleration was caused by missing material close to the edges and the resulting lower resistance of the flow. The edge flow can be simulated by adding a thin layer without porous media, but the goal of the simulation is avoiding the voids so simulation without the edge flow is safer.

It is planned in the further work to expand the simulations by the distribution layer. It should increase the speed of the resin in the mould and allow production of more complex parts. Further experiments will be carried out with a focus to automatize the image processing part to obtain reasonable results. 
"This publication was supported by the Czech Ministry of Industry and Trade in the framework of the institutional support for long-term conceptual development of research organization - recipient VÚTS, a.s."

\section{References}

1. A. A Ali, R. Umer, K. A Samad, Y. A Samad, 21st International Conference on Composite Materials (2017)

2. D. Gay, Composite materials design and applications (Taylor \& Francis Group, 2015) ISBN 978-1-4665-8488-4

3. Y. Hou, K. Taniguchi, L. Mailly, S. S. Wang, 27th Annual Conference of American Society of Composites (2012)

4. L. Nguyen, T. Juska, J. Mayes, 38th Structures, Structural Dynamics, and Materials Conference (1997), DOI: 10.2514/6.1997-1171

5. C. Lapoint, "CFD Modeling of the Flow of Resin into a Preform Mold of Carbon Fibers" (2015). Honors Theses. 347

6. A. B. Strong, Fundamentals of composites manufacturing (Society of Manufacturing Engineers) ISBN 0-87263-854-5

7. S. Abrate, Applied Mechanics Reviews, 55 (2002), DOI: 10.1115/1.1506323

8. Ansys Inc. ANSYS Fluent Theory Guide. Release 2019 R2 